\title{
A Rule Based Knowledge Transaction Model for Mobile Environments
}

\author{
Jianwen Chen ${ }^{1,2}$, Yan Zhang ${ }^{2}$ \\ ${ }^{1}$ IBM Global Service Australia, 20 Berry Street, North Sydney, NSW 2060, Australia \\ jchen@au1.ibm.com \\ ${ }^{2}$ School of Computing \& IT, University of Western Sydney, Penrith South DC NSW \\ 1797, Australia \\ yan@cit.uws.edu.au
}

\begin{abstract}
In this paper, we propose a rule based knowledge transaction model for mobile environments. Our model integrates the features of both mobile environment and intelligent agents. We use logic programming as a mathematic tool and formal specification method to study knowledge transaction in mobile environments. Our knowledge transaction model has two major advantages: (1) it has a declarative semantics inherited from logic programming; (2) it is also flexible in the sense that more complex knowledge transaction processes can be represented in our formalism. We show that our model can be used for knowledge transaction representation, formalization and knowledge reasoning in mobile environments. By illustrating a case study we demonstrate that our transaction model is applicable in practical domains in mobile environments.
\end{abstract}

Key words: knowledge transaction model, mobile environments, logic programming.

\section{Introduction}

Study on knowledge base and intelligent agent in mobile environments is a very new and meaningful research topic. As a practical scenario in this research area, a company manager may use mobile host to do the rule based decision making and negotiation. We believe that the investigation on intelligent agent and knowledge base in mobile environments is critical because this will help us to find a way to significantly improve current mobile system development. Comparing to the classical non-mobile environment, the mobile environment has a few specific properties such as mobility and disconnection. The issue of data and knowledge transactions has presented new challenges for researchers in mobile environments. Various proposals and systems have been developed in order to deal with database transaction processing in mobile environments $[1,5,13$, 18]. However, there seems to be a separation between multiagent systems and the intelligent agents community on one side, and the mobile agents community on the other side [14, 17, 22, 23]. Most of current research work concentrate on database not knowledge transaction of mobile environments $[1,5,13,18]$, and also these approaches suffer from a lack of formal specifications, which made these approaches be ac hoc for specific system and environment and could not be applied in general problem domains. As so far no any formal study has been conducted to the issue of knowledge transaction in mobile environment, as the first step, this paper addresses the accounts of knowledge transaction processing language and model in mobile environments. We develop a new knowledge transaction model for mobile environments, which integrates the features of 
both mobile environment $[1,5,18]$ and intelligent agents [22, 23]. Our knowledge transaction model is logic programming based, and has two major advantages: (1) our knowledge transaction model has a declarative semantics inherited from logic programming [3]; (2) Our knowledge transaction model is also flexible in the sense that more complex knowledge transaction processes can be formalized in our formalism. This model is rule based, and can be used for knowledge transaction representation, formalization and knowledge reasoning in mobile environments. We believe that our knowledge transaction language and model will provide a foundation towards the formal specification and development of real world mobile software systems, as the way of traditional software systems development. By illustrating a case study we demonstrate how our transaction model can be used in practical domains in mobile environments.

The paper is organized as follows. In Section 2, we present some background knowledge on intelligent agent and mobile environments, and then introduce our new environmental model. In section 3, we describe the transaction processing in mobile environments and give background knowledge on logic programming. Then we give mobile semantics to some logic programming concepts and formulas. In section 4, we start from knowledge transaction representation language, then impose a set of rules for knowledge transaction in mobile environments. Lastly we formalize our knowledge transaction model. In section 5 , we go through a transaction example to demonstrate how our knowledge transaction model can be used in practical scenario. Finally in section 6, we conclude and summarize our work.

\section{Environment Model}

To develop our knowledge transaction model in mobile environment, we propose a new environment model, which combines the features of mobile environments $[1,5,13,18]$ and intelligent agents $[22,23]$. This environment model can be used to study transaction processing [6,11], intelligent agent and knowledge base in mobile environment. In our paper, we use this model for knowledge transaction study in mobile environments.

When we study the transaction processing in mobile environments, we choose the environment model discussed in the paper $[1,5,18]$ to represent the salient features of mobile environments. We are looking at a Home Server (HS) acting as permanent storage of Mobile hosts' (MH) Files. There are Mobile Support Stations (MSS) providing services to a MH when it is within its cell. The MSS is connected to the HS via hardwires. The MH is continuously connected to a MSS via a radio link while accessing data. It may, however, become disconnected either voluntarily or involuntarily. When a $\mathrm{MH}$ registers with a MSS, a proxy is created on its behalf. It then performs various services for the $\mathrm{MH}$ including caching of profile, broadcasting of sub-profile, acquiring and releasing of locks and management of messages and page validations.

Home server (HS): permanent storage of the mobile host's files.

Mobile Support Station (MSS): provides services to MH (Mobile Host) such as caching, $\mathrm{RPC}$, etc. It also communicates with $\mathrm{MH}$ while it is within its cells. A MH is registered with a MSS when it enters into its range. The MSS is connected to the HS via hardwires. 
Connectivity: the MH is continuously connected to a MSS via a wireless link while accessing data. It may, however, become disconnected either voluntarily or involuntarily. Proxy: a proxy is created for a MH when it registers with a MSS. The proxy contacts the HS to obtain the profile of the MH. It then caches this profile on the MSS and broadcasts the sub-profile to the MH. It acquires and releases locks for the MH and manages its page invalidations and messages. The proxy buffers messages and invalidation until the $\mathrm{MH}$ is ready to receive them.

A typical transaction model in mobile environments can be described as shown in Figure 1:

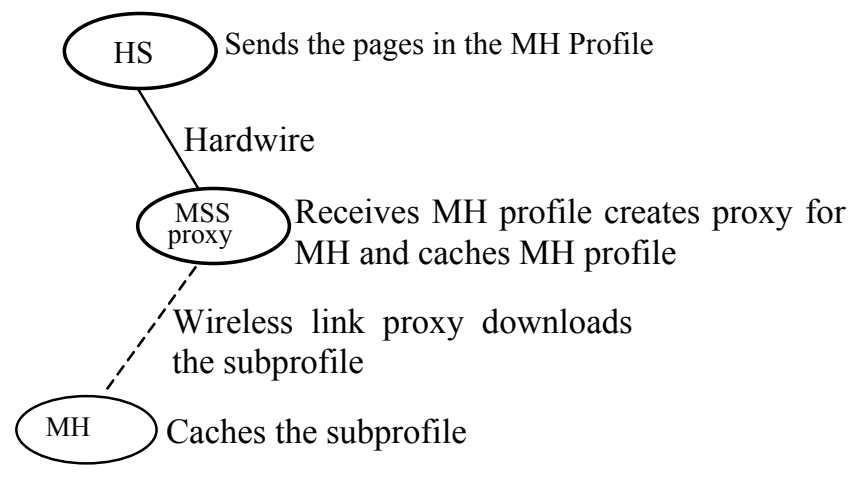

Figure. 1. Transaction Model in Mobile Environments

There is a centralized database residing in the HS. On each mobile host, $\mathrm{MH}$, there resides a transaction manager which preprocesses transaction operations; a scheduler which controls the relative order in which transaction operations are executed; a recovery manager which is responsible for commitment and abortion management and a cache manager. We assume that the effect of the scheduling is equivalent to an assignment of a local execution time. The MSS of the MH holds a local clock which synchronizes with this local execution time. The recovery manager updates the list of valid pages by having the MH contacting its proxy who then broadcasts an invalidation report. To have control over the problem of cell migration by the $\mathrm{MH}$, the HS holds a global clock against which all local clocks held by the MSS synchronize via some master-slave algorithm [16].

When we study the intelligent agent in the classical environment, we usually use the following environment model $[22,23]$ as shown in Figure 2.

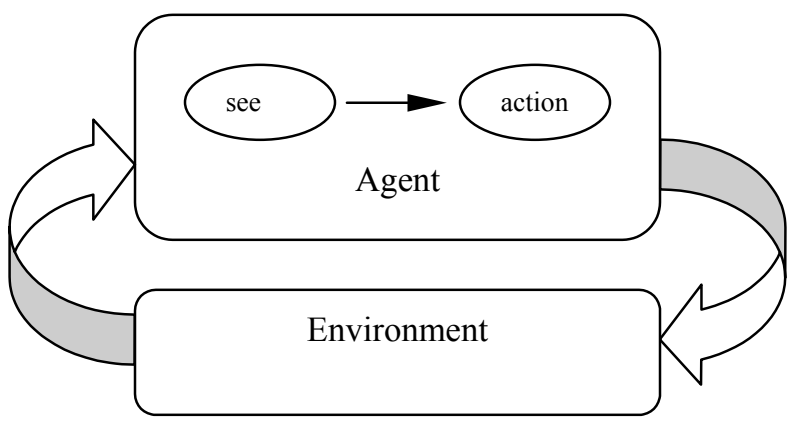

Figure 2. Intelligent Agent Model 
The idea is that function see captures the agent's ability to observe its environment whereas function action represents the agent's decision making process. Fundamentally, an agent is an active object with the ability to perceive, reason and act. We assume that an agent has explicitly represented knowledge and a mechanism for operating on or drawing inferences from its knowledge. We also assume that an agent has the ability to communicate. This ability consists of perception (the receiving of messages) and action (the sending of messages). In a distributed computing system, agents need to communicate with each other in order to achieve their goals. For this purpose, intelligent agent has been introduced for more flexible and efficient problem solving. We now propose a new environment model, which contains intelligent agents in mobile environment as shown in Figure 3.

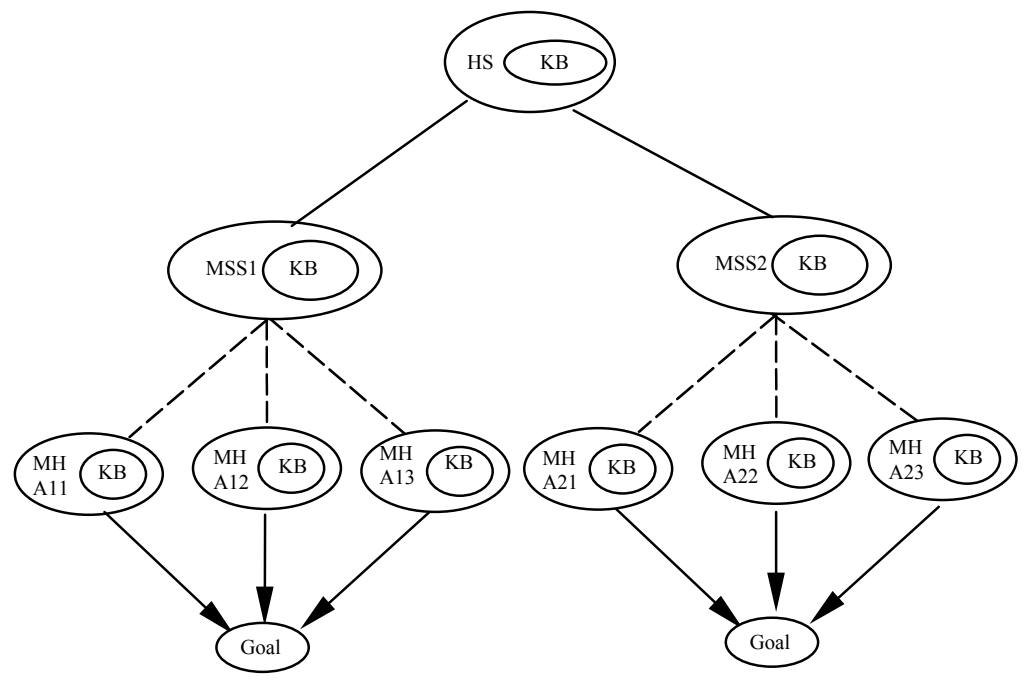

Figure 3. Intelligent Agent in Mobile Environment

In the environment model above, we assume that every Mobile Host $(\mathrm{MH})$ has its own knowledge base (KB) and intelligent agent (A11, A12, A13, A21, A22, A23), every MSS has knowledge base residing on it as well, MSS1 and MSS2 represent different MSS in different geographic areas. In Home Server (HS) level, there is a knowledge base that has a set of rules in it. Every intelligent agent on MH will work on behalf of $\mathrm{MH}$ that resides on, all the agents in the same geographic area (i.e. controlled by same HS) will negotiate, communicate, and cooperate with each other to achieve the goal for themselves and their systems. Such as agents can do decision making based on the rules in every knowledge base.

\section{Mobile Transaction Processing and Logic Programs}

\subsection{Transactions in Mobile Environments}

In this section, we will briefly describe the transaction processing in the mobile environment and give some background knowledge on logic programming, which will 
provide a basis for our logic programming based transaction processing language and model.

A typical transaction $T$ in the mobile environment will look like the following [7];

Contact-proxy $\rightarrow r[x] \rightarrow w[x] \rightarrow$ contact-proxy $\rightarrow i n v \rightarrow \ldots \rightarrow$ contact-proxy $\rightarrow s \rightarrow$ $w u \rightarrow \ldots \rightarrow c$

in which the proxy first acquires the appropriate set of locks for the MH. Once a write is executed, the proxy is asked to broadcast a report of invalidation to proclaim the existence of a new version of $x$. In this example, the $\mathrm{MH}$, after serving a number of operations of the $T$, decides to go to sleep voluntarily. This is done by first contacting the proxy and then flushing all its dirty pages (to the MSS) and releasing all the write-locks it holds. Thus the process of voluntary sleep is equivalent to a partial commit. It is a partial one because there might be operations still not executed.

Upon waking up, the $\mathrm{MH}$ goes through a process similar to start-up, i.e. there will be fetches of data objects (possibly through the proxy) and lock requests through the proxy. The remaining operations are then executed under the covering of the locks. Finally, the transaction is committed through delayed writes [18] to the MSS.

In the case of involuntary sleep the proxy is not contacted and therefore no invalidation report is broadcasted. Hence any update done by $\mathrm{MH}$ is lost. This is equivalent to there being another write to undo the previous write. If the $\mathrm{MH}$ holds write-locks while it goes to sleep involuntarily and if it does not wake up beyond a certain system prescribed time interval (as measured from the master clock of the HS), the write-locks are cancelled. Thus the overall effect is that of a partial abort. If the wake-up is soon enough, the locks are still with the $\mathrm{MH}$ and the execution of rest of the operations can proceed.

It is also possible for a transaction to be started with the $\mathrm{MH}$ being in one cell and completed with the $\mathrm{MH}$ in another one. In this case, the transaction $\mathrm{T}$ becomes

contact-proxy $\rightarrow r[x] \rightarrow w[x] \rightarrow$ contact-proxy $\rightarrow$ inv $\rightarrow \ldots \rightarrow$ move

$\rightarrow$ contact-proxy $\rightarrow \ldots \rightarrow c$

Here we have lumped all the activities related to handoff and the creation of a new proxy by a new MSS into the operation move.

\subsection{Extended Logic Programs: Syntax and Semantics}

In the non-mobile environment, traditional logic programming is used as a knowledge representation tool. An important limitation of this method is that logic programming does not allow us to deal directly with incomplete information, and therefore we only can get either yes or no answer from a query. This is because in the traditional logic programming, closed world assumption is automatically applied to all predicates [10], and each ground atom is assumed to be false if it does not been certified to be true in the 
program. The query evaluation methods of traditional logic programming give no answer to every query that does not succeed, it provides no counterpart for undecided situations which mean the incompleteness of information in classical axiomatic theories.

When we study knowledge transaction in mobile environments, we should clearly understand that there is a major different between the scenario that the transaction fails and the transaction hangs on due to mobile user's sleep. The first scenario is transaction fails in the sense of its negation succeeds, it is a no answer for a query. The second scenario is that transaction does not succeed because of incomplete information, the answer is unknown for a query transaction, but may become a definite answer yes or no after sometime. Therefore, in the mobile environment, we need a method which can deal with incomplete information explicitly, and this method should handle the transaction fails in the sense of its negation succeeds and the transaction that does not succeed in the mobile situation. The extended logic programs (ELPs) $[4,10,19,21]$ can overcome such limitation. It contains classical negation $\neg$ in addition to negation-as-failure not. Traditional logic programs provide negative information implicitly through closed-world reasoning, an extended logic program can include explicit negative information. In the language of extended programs, we can distinguish between a query which fails in the sense that it does not succeed and a query which fails in the stronger sense that its negation succeeds. By adding mobile semantics to extended logic programs, we can use this method to study knowledge transaction and deal with the incomplete information explicitly in the mobile environment. The applicability of extended logic programs for formalization of reasoning with incomplete information has been demonstrated in paper $[4,10,15,20]$.

Here, we give mobile semantics to some logic programming concepts and formulas in mobile environments, such as classical negation $\neg$, negation-as-failure not $[9,10]$, and closed world assumption [10]. The extended logic programs contain classical negation $\neg$, in addition to negation-as-failure not. In mobile semantics, classical negation $\neg$ is defined as explicit negative information, is explicit no when transaction is explicit fail. In the situation the mobile host is in voluntary or involuntary sleep, and therefore the information is incompleteness, we say it is absent of atom $A$, noted by not $A$, therefore it is unkown.

We consider closed world assumption from mobile semantics as following:

If mobile host is in voluntary or involuntary sleep and sleep time is beyond the limited time, we say we assume not $p$ as $\neg p$ at this time, unkown become no for the transaction. We can express closed world assumption by the rule

$\neg p(x) \leftarrow \operatorname{not} p(x), \quad T \leq x$

Here $\mathrm{T}$ is the time limit for mobile hosts' sleep.

A general logic program [10] can be defined as a set of rules of the form

$A_{0} \leftarrow A_{1}, \ldots, A_{m}$, not $A_{m+1}, \ldots$, not $A_{n}$, 
Where $0 \leq m \leq n$, and each $A_{i}$ is an atom.

An extended logic program is a set of rules of the form

$L_{0} \leftarrow L_{1}, \ldots, L_{m}$, not $L_{m+1}, \ldots$, not $L_{n}$,

Where $0 \leq m \leq n$, and each $L_{i}$ is a literal. A literal is a formula of the form $A$ or $\neg A$, where $\mathrm{A}$ is an atom. The negation sign in the negative literal $\neg A$ represents classical negation, not negation-as-failure, so that expression of the form not $A$, occurring in general logic program, is not literal according to this definition.

The answer set of an extended logic program is normally defined as follows [4];

Let $\Pi$ be an extended program without variables that doesn't contain not, and let Lit be the set of ground literals in the language of $\Pi$. The answer set of $\Pi$ is the smallest subset $S$ of Lit such that

(i) for any rule $L_{0} \leftarrow L_{1}, \ldots, L_{m}$ from II, if $L_{1}, \ldots, L_{m} \in S$, then $L_{0} \in S$;

(ii) if $S$ contains a pair of complementary literals, then $S=$ Lit.

The answer set of a program $\prod$ that doesn't contain negation-as-failure can be denoted by $\alpha(\Pi)$.

We say $\Pi$ entails a literal $L$, denoted as $\Pi \mid=L$, if $L$ is true in all answer sets of $\Pi$.

In our logic programming based transaction language and model, logic programming is used as a formal specification method to describe both static and dynamic transaction processing.

\section{A Logic Programming Based Transaction Model}

In this section, we define a logic programming based knowledge transaction model which formalizes the knowledge transaction processing in mobile environments. We start with a complete transaction stage by stage including startup, sleep, wakeup, move/handoff, read, write and commit, to give readers a clear idea what activities are supposed to happen on $\mathrm{MH}, \mathrm{MSS}$ and HS at every stage. Then we define a transaction processing language $\mathscr{L}$, which contains necessary components for specifying knowledge transactions associated with $\mathrm{MH}, \mathrm{MSS}$ and HS. Finally, we use language $\mathscr{L}$ to specify a knowledge transaction model that imposes all logic rules to capture transaction features in our mobile environment.

\subsection{Transaction Processing at Three Levels}

Let us go over a transaction processing stage by stage firstly to see what activities happen on MH, MSS and HS on every transaction stage [18]. Here, we specify the activities for transaction start and commit as well. 
Startup is the initial powering up of the mobile computer, after registration the $\mathrm{MH}$ can start a query or update transaction. Voluntary or involuntary sleep can happen in the transaction. After the sleep, the MH can wake up to continue the transaction. Also, the MH can start the transaction in one MSS cell and move to another MSS cell to continue the transaction. The last activity of transaction is transaction commit or abort. We show all the possibilities of transaction activity in the Figure 4. The transaction activity can be: startup $\rightarrow$ start $\rightarrow$ sleep $\rightarrow$ wakeup $\rightarrow$ commit; Or startup $\rightarrow$ start $\rightarrow$ move $\rightarrow$ commit. The transaction can follow the arrow in the figure to process the transaction activities.

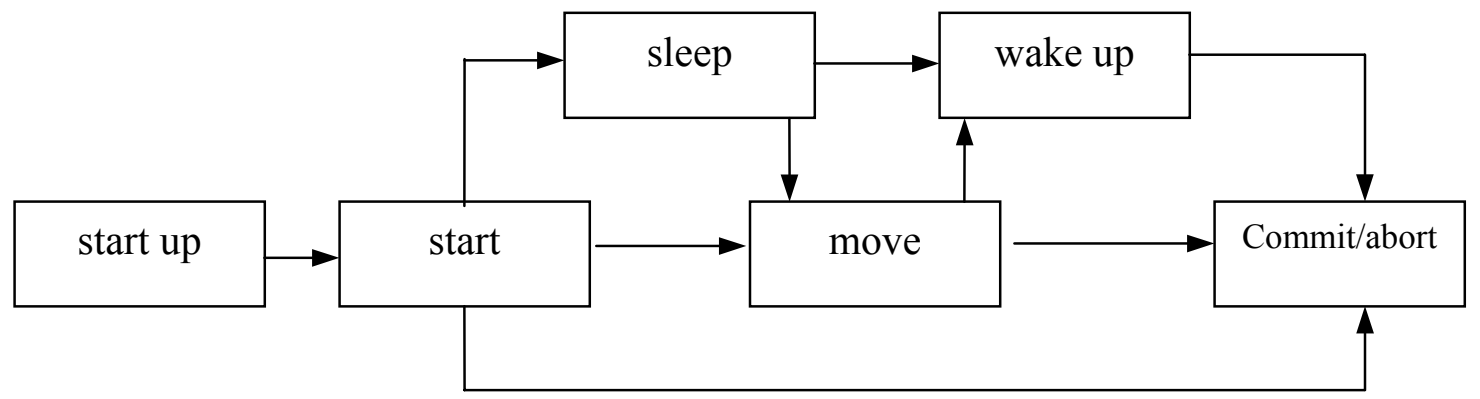

Figure 4. Transaction Activities in Mobile Environments

There are different steps are involved on every stage of the transaction;

- Startup

Startup is the initial powering up of the mobile computer. The following are the steps involved in starting up the $\mathrm{MH}$ :

Step 1: When the MH is powered on, it registers with MSS. Upon registration, the MH notifies the MSS of its HS address.

Step 2: MSS then creates the proxy process which retrieves the MH profile from HS.

Step 3: HS sends the pages in the $\mathrm{MH}$ profile, marks the $\mathrm{MH}$ as a valid reader of those pages, and notes where to contact the $\mathrm{MH}$.

Step 4: The proxy receives and caches the MH profile.

Step5: The proxy then broadcasts the sub-profile to the $\mathrm{MH}$, and marks the in-MH-cache bit for those pages

Step 6: The MH receives and caches the sub-profile pages.

- Start Transaction

Step1: The MH requests a query or update transaction.

Step 2: The MH acquires a lock if it is an update transaction.

Step 3: The MSS submits the transaction request to HS.

Step 4:The HS does the transaction after MSS submits the transaction request.

- Sleep 
There are essentially two types of sleep - voluntary and involuntary. Voluntary sleep is a planned power down, while involuntary sleep is an unplanned power down, i.e., the system crash or run out of battery power.

Voluntary Sleep:

Step1: The MH flushes its dirty pages, gives up any write locks it holds, and informs the proxy of its intention to sleep.

Step 2: The proxy updates MH-sleep-time and buffers messages and invalidations for the $\mathrm{MH}$ until the MH wakes up and is ready to receive them.

Involuntary Sleep:

In this case, the proxy does not know that $\mathrm{MH}$ is not listening and continues to broadcast invalidations as normal. The $\mathrm{MH}$ will recover missed messages upon wakeup by asking for all messages sent after its disconnection time. Suppose the MH is holding a write lock when it goes to sleep involuntarily and in the meanwhile, another writer may ask HS for the write lock. HS forwards the request to the proxy, and the proxy forwards it to the MH. If proxy does not receive the lock from the $\mathrm{MH}$ in a limited amount of time, it invalidates the lock and sends it back to HS. The MH will inevitably lose the updates it had made.

One potential problem is that the sleeping $\mathrm{MH}$ process may not return (e.g., MH dies, leaves cell), in which case the proxy may wait around aimlessly. To remedy this problem, the MH state is sent to HS after a system-specific amount of time and the proxy process is killed (the decision is made based on MH-sleep-time or time-MH-contacted-proxy). Should the MH return, the wakeup is treated as a startup process.

- Wakeup

Wakeup is the powering up after a MH has been asleep. Although similar to startup, wakeup has slightly different semantics. The wakeup sequence is as follows:

Step1: Upon wakeup, the MH waits to get MSS's address (beacon).

Step 2: Upon receiving the beacon, the MH sends wakeup notification to MSS and requests missed messages. If the $\mathrm{MH}$ has saved its $\mathrm{MH}$-sleep-time (possible in the case of voluntary sleep), it sends this information to the MSS. Otherwise. If the MH had gone to sleep involuntarily (e.g., power failure), the MSS proxy uses time-MH-contacted-proxy and time-invalidation-propagated for each page on its cache to calculate how many old messages should be resent.

\section{- Move/Handoff}

Step 1:The MH listens for MSS beacon.

Step 2: When the MH notices that it is in a different region, it contacts the new MSS. The message sent includes the MH's id, MH's HS, MH's old MSS, and last-time-MSScontacted-MH (for the old MSS). 
Step 3: The new MSS contacts the old MSS to get the state of the MH proxy.

Step 4: The old MSS flushes any dirty pages to HS and sends the proxy state to the new MSS.

Step 5: The new MSS proxy contacts HS to tell it where to contact the MH.

Step 6: The new MSS proxy broadcasts any invalidations whose timestamp is later than last-time-MSS-contacted-MH.

- Commit Transaction

Step 1: The HS decides if the transaction should be committed or aborted according to the two phase commit protocol, then commits or aborts the transaction.

Step 2: The HS sends the transaction result to MSS

Step 3: The MSS broadcasts the transaction result to $\mathrm{MH}$.

Step 4: The MH updates the local knowledge base according to the transaction result.

\subsection{Logic Programming Formalizations I: The Language $\mathscr{L}$}

The syntax of the language $\mathscr{L}$ contains variables of three sorts: situation variable $s$, fluent variable $f$, and action variable $a$. Language $\mathscr{L}$ has one predicate $\operatorname{holds}(f, s)$, where $f$ is a fluent function, and $s$ is a situation. The predicate $\operatorname{holds}(f, s)$ means that fluent $f$ is true at situation $s$. The term fluent is introduced in paper [2] to represent predicates and functions whose value changes over time. $\mathscr{L}$ also has a resulting function $\operatorname{res}(a, s)$, which denotes a situation resulting from situation $s$ by performing action $a$. To begin with, $\mathscr{L}$ has one initial situation constant $S_{0}$. There are also some other predicates and function symbols. The sorts of their arguments and values will be clear from their use in the rules.

Now we go over an example to show how to represent knowledge in logic programs. We are given a complete description of the initial state of the world and a complete description of the effects of actions, and we are asked to determine what the world will look like after a series of actions is performed [4]. The most frequently cited example of such reasoning is probably the Yale Shooting Problem (YSP) from [12]. In the Yale Shooting Problem [4], there are two fluents: alive and loaded, and three actions: wait, load, and shoot. We know that the execution of loading leads to the gun being loaded, and that if the gun is shot while it is loaded, a turkey Fred dies. We want to predict that after the execution of actions load, wait, and shoot (in that order), Fred will be dead. It seems that the commonsense argument which leads to this conclusion is based on the so called axiom of inertia which says, "Things normally tend to stay the same". As a typical normative statement, it can be represented by the rule

y1: holds $(F, \operatorname{res}(A, S)) \leftarrow \operatorname{holds}(F, S), \operatorname{not} a b(y 1, A, F, S)$

To represent the effect of the actions load, shoot, and wait, we need only the rule y2: holds(loaded, res(load, S)) $\leftarrow$ 
And the cancellation rule

y3: ab(y1, shoot, alive, S) $\leftarrow$ holds (loaded, S)

Which represent the priority of specific knowledge about the results of actions over the general law of inertia. Let $S_{0}$ be the initial state, and suppose we are given that

y4: holds(alive, $\left.S_{0}\right)$

Even though the resulting program $\gamma$ consisting of $y 1-y 4$ is not stratified, it is possible to show that it has a unique stable model. It is easy to see that $\gamma$ entails

holds(alive, $\left.\operatorname{res}\left(\operatorname{load}_{,} S_{0}\right)\right)$, and

$\neg$ holds(alive, res(shoot, res(wait, res $\left(\right.$ load, $\left.\left.\left.S_{0}\right)\right)\right)$ )

Here, we give another knowledge representation example to discuss a very simple knowledge base update transaction. We have only one fluent: updated, and two action: commit and abort. We know that commit will result in the knowledge update. We want to predict that after the execution of action commit, knowledge base will be updated.

K1: holds $(F, \operatorname{res}(A, S)) \leftarrow \operatorname{holds}(F, S), \operatorname{not} a b(K 1, A, F, S)$

To represent the effect of the action commit, we need only the rule

\section{K2: holds(updated, res(commit, S)) $\leftarrow$}

If the transaction is abort, the knowledge base won't be updated. So we have cancellation rule

K3: ab(K1, abort, updated, $S) \leftarrow$

In our rule based knowledge transaction model, we start to define a transaction processing language $\mathscr{L}$ to formalize transaction related action and fluent function at $\mathrm{MH}$, MSS and HS level. We first introduce different sorts of functions to characterize the basic components of our language. We use actions and fluents to denote transaction processing activities, results and status. Then $\mathscr{L}$ has the following specific action and fluent functions at different level.

- Mobile Host (MH) level

We have the following action functions:

Move $(y, x)$ : denotes MH $x$ moves into MSS $y$ cell.

Query(x): denotes $\mathrm{MH} x$ has a query transaction request.

Write $(x)$ : denotes $\mathrm{MH} x$ has a update transaction request. 
Acquire-lock(x): denotes $\mathrm{MH} x$ requests a lock.

Flush $(x)$ : denotes MH $x$ flushes its dirty pages.

Release-lock $(x)$ :denotes $\mathrm{MH} x$ releases its write lock.

Request-message (x): denotes MH $x$ requests missed message from MSS $y$.

Fetch-message $(x)$ : denotes MH $x$ fetches missed message from MSS $y$.

Update-knowledge $(x)$ : denotes $\mathrm{MH} x$ updates local knowledge base according to the transaction result.

We have the following fluent functions:

Registered $(y 1, x)$ : denotes $\mathrm{MH} x$ has registered in MSS1 $y 1$.

Query-requested $(x)$ : denotes $\mathrm{MH} x$ has requested to start a query transaction.

Trans-start $(x)$ : denotes $\mathrm{MH} x$ has started a transaction.

Update-requested $(x)$ : denotes $\mathrm{MH} x$ has requested to start a update transaction.

Locked $(x)$ : denotes $\mathrm{MH} x$ has got a lock for a update transaction.

Vol-slept $(x)$ : denotes $\mathrm{MH} x$ has gone to voluntary sleep.

Sleep-sig $(x)$ : denotes there is a sleep signal on $\mathrm{MH} x$.

Invol-slept $(x)$ : denotes $\mathrm{MH} x$ has gone to involuntary sleep.

Lock-cancelled $(x)$ : denotes write lock has been cancelled on $\mathrm{MH} x$.

Update-lost $(x)$ : denotes updates have been lost on $\mathrm{MH} x$.

Wakeup-sig(x): denotes there is a wakeup signal on $\mathrm{MH} x$.

Message-received $(x)$ : denotes $\mathrm{MH} x$ has received missed messages.

Registered $(y 2, x)$ : denotes $\mathrm{MH} x$ has registered in MSS2 y2.

Knowledge-updated $(x)$ : denotes MH $x$ has updated the local knowledge base.

Commit-broadcasting $(y, x)$ : denotes MSS $y$ has noticed transaction commit to MH $x$.

Abort-broadcasting $(y, x)$ : denotes MSS $y$ has noticed transaction abort to $\mathrm{MH} x$.

Timeoutl(x): denotes $\mathrm{MH} x$ has expired a certain time1.

- Mobile Support Station (MSS) level

We have the following action functions:

Create-proxy $(y 1, x)$ : denotes MSS1 $y 1$ creates proxy process for MH $x$.

Create-proxy $(y 2, x)$ : denotes MSS2 $y 2$ creates proxy process for MH $x$.

Retrieve (y): denotes MSS $y$ proxy retrieves the MH $x$ profile from HS.

Cache(y): denotes MSS $y$ proxy caches the MH profile.

Broadcast(y): denotes MSS $y$ proxy broadcasts the sub-profile to the MH.

$\operatorname{Mark}(y)$ : denotes MSS $y$ proxy marks the in-MH-cache bit for those broadcasting pages.

Submit(y): denotes the transaction has been submitted to HS by MSS $y$.

Update-sleeptime (y): denotes MSS $y$ proxy updates MH-sleep-time.

Buffer $(y)$ : denotes MSS $y$ proxy buffers the message and invalidations for MH until the MH wakes up.

Page-broadcast $(y 1, x)$ : denotes MSS1 $y 1$ proxy does the page broadcasting to MH $x$.

Page-broadcast $(y 2, x)$ : denotes MSS2 $y 2$ proxy does the page broadcasting to MH $x$.

Cancel-lock(y): denotes MSS $y$ proxy cancels the lock for transaction.

Flush(y1): denotes MSS1 yl flushes any dirty pages to HS. 
$\operatorname{Broadcast}(y, x)$ : denotes MSS $y$ broadcasts the transaction result to $\mathrm{MH} x$.

We have the following fluent functions:

$\operatorname{Proxy}(y 1, x)$ : denotes MSS $1 y 1$ has created proxy process for $\mathrm{MH} x$. $\operatorname{Proxy}(y 2, x)$ : denotes MSS2 $y 2$ has created proxy process for $\mathrm{MH} x$.

Cached(y): denotes MSS y proxy has cached the MH profile.

Broadcasting $(y)$ : denotes MSS $y$ proxy has done the sub-profile broadcasting to $\mathrm{MH}$.

Marked(y): denotes MSS $y$ proxy has marked in-MH-cache bit for pages.

Trans-submitted(y): denotes that the transaction has been submitted by MSS $y$.

Buffered(y): denotes MSS y proxy has buffered messages and invalidations for $\mathrm{MH}$.

Page-broadcasting(y): denotes MSS $y$ proxy has done the page broadcasting.

Timeout $(x)$ : denotes involuntary sleep time has expired on $\mathrm{MH} x$.

Move-sig(y2): denotes MH has moved to MSS2 y2.

Flushed(y1): denotes MSS1 yl has flushed any dirty pages to HS.

Commit-broadcasting $(y, x)$ : denotes MSS $y$ has noticed transaction committing to $\mathrm{MH} x$.

Abort-broadcasting $(y, x)$ : denotes MSS $y$ has noticed transaction aborting to MH $x$.

Commit-noticed $(z, y)$ : denotes HS $z$ has given transaction commit notice to MSS $y$.

Abort-noticed $(z, y)$ : denotes HS $z$ has given transaction abort notice to MSS $y$.

\section{- Home Server (HS) level}

We have the following action functions:

Send-page $(z, y)$ : denotes HS $z$ sends the pages to MSS $y$ in the MH profile.

$\operatorname{Mark}-M H(z)$ : denotes HS $z$ marks the MH as a valid reader of those pages.

Do-trans(z): denotes HS $z$ does the transaction.

$\operatorname{Kill}-\operatorname{proxy}(z, y)$ : denotes HS $z$ kills the MSS $y$ proxy process.

Send-dirtypage $(z, y 2)$ : denotes HS $z$ sends any dirty pages to new MSS2 $y 2$.

Commit( $z)$ : denotes HS $z$ commits the transaction.

Notice-commit $(z, y)$ : denotes HS $z$ gives transaction commit notice to MSS $y$.

Notice-abort $(z, y)$ : denotes HS $z$ gives transaction abort notice to MSS $y$.

We have the following fluent functions:

$\operatorname{Sent}(z, y)$ : denotes HS $z$ has sent pages to MSS $y$.

MH-marked(z): denotes HS $z$ has marked the MH as a valid reader for those pages.

Trans-started(z): denotes HS $z$ has started transaction processing.

Proxy-killed $(z, y)$ : denotes HS $z$ has killed MSS $y$ proxy process for MH.

Trans-committed(z): denotes transaction has been committed on HS $z$.

Commit-agreed (z): denotes HS $z$ has got transaction commit agreement.

Abort-agreed (z): denotes HS $z$ has got transaction abort agreement.

Timeout2(z): denotes HS $z$ has expired a certain time2.

Commit-noticed $(z, y)$ : denotes HS $z$ has given transaction commit notice to MSS $y$. 
Abort-noticed $(z, y)$ : denotes HS $z$ has given transaction abort notice to MSS $y$.

\subsection{Logic Programming Formalizations II: Modeling}

Based on the transaction processing language $\mathscr{L}$ defined above, we start to specify and impose all necessary rules to formalize a logic programming based transaction processing model in mobile environment, which models all transaction processing activities, requests, results and constraints on MH, MSS, and HS three levels.

\section{- Mobile Host (MH) level}

\section{Register}

When MH moves into MSS cell, it is registered. The rule for this is as following:

rl: holds(registered $(y 1, x)$, res(move $(y 1, x), s)) \leftarrow$

After an action move $(y 1, x)$ happens, $\operatorname{res}(\operatorname{move}(y 1, x), s)$ becomes the current situation, and registered $(y 1, x)$ is true.

\section{Start a query or update transaction}

For a query transaction, as long as $\mathrm{MH}$ has a transaction request, the transaction should be started straight away. The rules are as following:

$$
\text { r2: holds(query-requested }(x) \text {, res(query }(x), s)) \leftarrow
$$

After an action query $(x)$, i.e., the MH submits a query request, $\operatorname{res}(q u e r y(x), s)$ becomes the current situation, and query-requested $(x)$ becomes true.

$$
\text { r3: holds(trans-start( }(x), s) \leftarrow \text { holds(query-requested( }(x), s)
$$

After query-requested $(x)$ becomes true, $\operatorname{trans-start}(x)$ is true for a query transaction.

For an update transaction, after MH has a write request, the lock need to acquired firstly to start this transaction. The transaction will start after the lock is available.

$$
\text { r4: holds(update-requested (x), res(write( }(x), s)) \leftarrow
$$

After an action write $(x)$, i.e., the MH submits a write request, res(write $(x), s)$ becomes the current situation, and update-requested $(x)$ becomes true.

$$
\text { r5: holds(trans-start }(x), s) \quad \begin{aligned}
\leftarrow \text { holds(locked }(x), \text { res(acquire-lock }(x), \text { res(write }(x), s))), \\
\text { holds(update-requested }(x), s)
\end{aligned}
$$

After an action write $(x)$, another action acquire-lock $(x)$ takes place, res(acquire-lock $(x)$, res(write $(x), s)$ ) becomes current situation, locked $(x)$ becomes true, i.e., the $\mathrm{MH}$ gets a lock. If both locked $(x)$ and update-requested $(x)$ are true, trans-start $(x)$ is true as well, i.e., a update transaction starts.

\section{Sleep}

For a voluntary sleep, the MH informs the proxy of its intention to sleep, and then flushes its dirty pages, gives up any write-locks it holds, after these, the MH goes to voluntary sleep.

r6: holds(vol-slept(x), res(release-lock(x), res(flush(x), s))) $\leftarrow$ holds(sleep-sig $(x), s)$ 
After sleep-sig $(x)$ becomes true, action $f l u s h(x)$ and release-lock $(x)$ are taken place, and vol-slept $(x)$ becomes true eventually, i.e., the $\mathrm{MH}$ is in voluntary sleep.

For an involuntary sleep, we suppose that the $\mathrm{MH}$ is holding a write-lock when it goes to involuntary sleep and in the meantime, lock is asked by another writer. HS forwards the request to the proxy, and the proxy forwards it to the $\mathrm{MH}$. If proxy does not receive the lock from the $\mathrm{MH}$ in a limited amount of time, it invalidates the lock and sends it back to HS. And therefore, the MH will inevitably lose the updates it had made.

$$
\text { r7: holds(invol-slept }(x), s) \leftarrow
$$

The invol-slept( $x)$ is true, i.e., the $\mathrm{MH}$ is in involuntary sleep.

r8: holds(lock-cancelled $(x), s) \leftarrow$ holds(invol-slept $(x), s)$, holds(timeout $(x), s)$

If both invol-slept $(x)$ and timeout $(x)$ are true, lock-cancelled $(x)$ will become true as well, i.e., if the $\mathrm{MH}$ is in involuntary sleep and doesn't response to proxy in a period limit time, then the lock is cancelled on the $\mathrm{MH}$.

$$
\text { r9: holds(update-lost }(x), s) \leftarrow \text { holds(lock-cancelled( } x \text { ), s) }
$$

After lock-cancelled $(x)$ becomes true, update-lost $(x)$ becomes true as well, i.e., the $\mathrm{MH}$ loses all its update.

\section{Wake up}

Upon the MH waking up, the MH sends wakeup notification to MSS and requests missed messages. If the $\mathrm{MH}$ has saved its $\mathrm{MH}$-sleep-time, it sends this information to MSS. If the $\mathrm{MH}$ wakes up from involuntary sleep, the MSS proxy will use time-MH-contactedproxy and time-invalidation-propagated for each page on its cache to calculate how many old messages should be resent.

r10: holds(message-received(x), res(fetch-message( $x$ ), res(request-message $(x), s))) \leftarrow$ holds(wakeup-sig $(x), s)$

After wakeup-sig $(x)$ becomes true, the $\mathrm{MH}$ has action request-message $(x)$ and fetchmessage(x), then res(fetch-message $(x)$, res(request-message $(x), s)$ ) becomes current situation, then message-received $(x)$ is true on $\mathrm{MH}$.

\section{Move/handoff}

When the $\mathrm{MH}$ notices that it is in a different region, it contacts the new MSS. The message sent includes the MH's id, MH's HS, MH's old MSS and last-time-MSScontacted-MH for old MSS. After new MSS contact HS and old MSS, the MSS proxy broadcasts any invalidations whose timestamp is later than last-time-MSS-contacted-MH. r11: holds(registered $(y 2, x), s) \leftarrow \operatorname{holds}(\operatorname{move}-\operatorname{sig}(y 2, x)$, res(move $(y 2, x), s)$ ) After action move $(y 2, x)$ on $\mathrm{MH}$, move-sig $(y 2, x)$ becomes true, and then registered $(y 2, x)$ becomes true as well, which means the MH moves and registers in new MSS.

r12: holds (message-received $(y 2, x), s) \leftarrow \operatorname{holds}($ registered $(y 2, x), s)$

After registered $(y 2, x)$ is true, message-received $(y 2, x)$ becomes true as well, the $\mathrm{MH}$ receives necessary messages from new MSS.

\section{Transaction Commit}

After $\mathrm{MH}$ requests a transaction, the transaction will be committed or aborted on the HS according to the two phase commit protocol [6]. After that HS sends transaction result to MSS, the MSS broadcasts the transaction result to $\mathrm{MH}$, the $\mathrm{MH}$ updates the local knowledge base accordingly based on transaction commit or abort. If after transaction 
started for a period time (timeout1), the $\mathrm{MH}$ host still has not got any transaction commit or abort notice from MSS for whatever reason, we use closed world assumption in this case, assume not $\mathrm{p}$ as $\neg p$ at this time, unkown (not) becomes no ( $\neg$ ) for the transaction.

$$
\text { r13: holds(knowledge-updated(x), res(update-knowledge }(x), s)) \leftarrow
$$

holds(commit-broadcasting $(y, x), s)$, holds(trans-start(x), s)

If both commit-broadcasting $(y, x)$ and trans-start $(x)$ are true, then action updateknowledge $(x)$ happens, knowledge-updated $(x)$ becomes true.

$$
\begin{gathered}
\text { r14: } \neg \text { holds (knowledge-updated }(x), s) \leftarrow \\
\text { holds(abort-broadcasting }(y, x), s), \text { holds(trans-start }(x), s)
\end{gathered}
$$

If both abort-broadcasting $(y, x)$ and trans-start $(x)$ are true, then we know knowledgeupdated $(x)$ is not true.

$$
\text { r15: } \neg \text { holds(knowledge-updated }(x), s) \leftarrow \text { holds(trans-start }(x), s) \text {,holds(timeout1 }(x), s) \text {, }
$$

not holds(commit-broadcasting $(y, x)$, s), not holds(abort-broadcasting $(y, x), s$ )

If neither commit-broadcasting $(y, x)$ nor abort-broadcasting $(y, x)$ is holds (true), and time is over the limit, i.e., timeoutl ( $x$ ) is holds, then we know knowledge-updated $(x), s)$ is not true, it is false.

\section{- Mobile Support Station (MSS) level}

\section{Register}

After the MH registers with MSS, MSS creates the proxy process which retrieves the MH profile from HS, the proxy receives and caches the MH profile, and then broadcasts the sub-profile to the $\mathrm{MH}$, and marks the in-MH-cache bit for those pages. The rules for these are as following:

$$
\text { rl: holds }(\operatorname{proxy}(y, x), \operatorname{res}(\operatorname{create}-\operatorname{proxy}(y, x), s)) \leftarrow \operatorname{holds}(\text { registered }(y, x), s)
$$

After registered $(y, x)$ becomes true, the action create-proxy $(y, x)$ happens, res(create$\operatorname{proxy}(y, x), s)$ becomes current situation, then $\operatorname{proxy}(y, x)$ is in holds, which means MSS creates a proxy for the $\mathrm{MH}$.

$r 2: \operatorname{holds}(\operatorname{cached}(y), \operatorname{res}(\operatorname{cach}(y), \operatorname{res}(\operatorname{retrieve}(y), s))) \leftarrow \operatorname{holds}(\operatorname{proxy}(y, x), s)$ After $\operatorname{proxy}(y, x)$ becomes true, action retrieve $(y)$ and cache $(y)$ take place, then cached $(y)$ is true, i.e., MSS has cached for MH.

r3: holds(broadcasting $(y, x)$, res(broadcast $(y, x), s)) \leftarrow \operatorname{holds}(\operatorname{cached}(y, x), s)$

After cached $(y, x)$ becomes true, action broadcast $(y)$ takes place, then broadcasting $(y)$ is true.

$$
\text { r4: holds(marked(y), res(mark(y), s)) } \leftarrow \operatorname{holds}(\operatorname{broadcasting}(y, x), s)
$$

After broadcasting (y) becomes true, action mark(y) happens, then marked(y) becomes true, i.e., the MSS has marked broadcasting pages for $\mathrm{MH}$.

\section{Start a query or update transaction}

After $\mathrm{MH}$ requests a query or update, MSS submits this transaction request to HS on behalf of $\mathrm{MH}$. If it is a write request, the lock needs to be acquired firstly to submit this transaction.

$$
\text { r5: holds(trans-submitted(y), res(submit (y), s)) } \leftarrow \text { holds(query-requested( } x \text { ), s) }
$$


After query-requested(x) becomes true, action submit(y) happens, res(submit( $y)$, s) becomes current situation, trans-submitted(y) then becomes true.

r6: holds(trans-submitted(y), res(submit(y), s)) $\leftarrow$

holds(locked(x), s),holds(update-required(x), s))

If both update-required $(x)$ and locked $(x)$ are true, action submit(y) will happen and transsubmitted(y) then becomes true.

\section{Sleep}

For the voluntary sleep, the proxy updates MH-sleep-time and buffers messages and invalidations for the $\mathrm{MH}$ until the $\mathrm{MH}$ wakes up and is ready to receive them.

$$
\begin{aligned}
\text { r7: holds(buffered(y), } & \text { res(buffer(y), res(update-sleeptime(y), s))) } \leftarrow \\
& \text { holds(vol-slept }(y, x), s)
\end{aligned}
$$

After vol-slept $(y, x)$ becomes true, action update-sleeptime(y) and buffer $(y)$ will happen continuously and then res(buffer(y), res(update-sleeptime(y), s)) become the current situation, buffered(y) becomes true.

In the involuntary sleep case, the proxy doesn't know that $\mathrm{MH}$ is not listening and continues to broadcast invalidations as normal. If the $\mathrm{MH}$ is holding a write-lock when it goes to involuntary sleep, in the meantime the lock is asked by another writer, the proxy forwards this request to the $\mathrm{MH}$. If proxy does not receive the lock from the $\mathrm{MH}$ in a limited amount of time, it invalidates the lock and sends it back to HS.

r8: holds(page-broadcasting $(y, x)$, res(page-broadcast $(y, x), s)) \leftarrow$

In the involuntary sleep case, action page-broadcast $(y, x)$ still happens, pagebroadcasting $(y, x)$ is still true.

r9: holds(lock-cancelled(y, x), res(cancel-lock $(y, x), s)) \leftarrow$ holds(invol-slept(x), s), holds(timeout $(x), s))$

If both invol-slept(x) and timeout(x) are true, action cancel-lock $(y, x)$ will happen, i.e., proxy will cancel the lock for $\mathrm{MH}$ and then lock-cancelled $(y, x)$ becomes true.

\section{Wake up}

Upon the MH waking up, the MH sends wakeup notification to MSS and requests missed messages. If the MH has saved its MH-sleep-time, it sends this information to MSS. If the $\mathrm{MH}$ wakes up from involuntary sleep, the MSS proxy will use time-MH-contactedproxy and time-invalidation-propagated for each page on its cache to calculate how many old messages should be resent.

r10: holds(page-broadcasting $(y, x)$, res(page-broadcast $(y, x), s)) \leftarrow$ holds(wakeup-sig(x), s)

If wakeup-sig $(x)$ is true, action page-broadcast $(y, x)$ will take place, then pagebroadcasting $(y, x)$ becomes true.

\section{Move/handoff}

After MH contacts and registers in the new MSS, the new MSS contacts the old MSS to get the MH proxy status. The old MSS flushes any dirty pages to HS and sends the proxy status to the new MSS. The new MSS proxy contacts HS to tell it where to contact the MH. The new MSS proxy broadcasts any invalidations whose timestamp is later than last-time-MSS-contacted-MH.

$$
\text { r11: holds(proxy }(y 2, x) \text {, res(create-proxy }(y 2, x), s)) \leftarrow \operatorname{holds}(\operatorname{registered}(y 2, x), s)
$$


After registered $(y 2, x)$ becomes true, i.e., $\mathrm{MH}$ has registered in new MSS, action create$\operatorname{proxy}(y 2, x)$ happens, and then $\operatorname{proxy}(y 2, x)$ is true.

r12: holds(flushed(y1), res(flush(y1), s)) $\leftarrow \operatorname{holds}(\operatorname{move-sig}(y 2, x), s)$

After move-sig $(y 2, x)$ becomes true, action flush(y1) happens and then flushed(y1) becomes true, i.e., old MSS flushes dirty pages for $\mathrm{MH}$.

r13: holds(page-broadcasting $(y 2, x)$, res(page-broadcast $(y 2, x), s)) \leftarrow$ holds $(\operatorname{proxy}(y 2, x), s)$

After $\operatorname{proxy}(y 2, x)$ becomes true, i.e., new MSS has created proxy for $\mathrm{MH}$, action pagebroadcast $(y 2, x)$ happens, ), res(page-broadcast $(y 2, x), s)$ becomes current situation, page-broadcasting $(y 2, x)$ then becomes true.

\section{Transaction Commit}

After MSS gets the transaction commit or abort notice from HS, the MSS will broadcast the transaction result to $\mathrm{MH}$ accordingly.

r14: holds(commit-broadcasting $(y, x)$, res(broadcast $(y, x), s)) \leftarrow$ holds(commit-noticed $(z, y), s)$

If commit-noticed $(z, y)$ is true, i.e., MSS has got commit notice from HS, action broadcast $(y, x)$ will happen, commit-broadcasting $(y, x)$ then becomes true, which means MSS will sent commit broadcasting to $\mathrm{MH}$.

$$
\begin{gathered}
\text { r15: holds(abort-broadcasting }(y, x), \operatorname{res}(\operatorname{broadcast}(y, x), s)) \leftarrow \\
\text { holds(abort-noticed }(z, y), s)
\end{gathered}
$$

If abort-noticed $(z, y)$ is true, i.e., MSS has got abort notice from HS, action broadcast(y, $x$ ) will happen, abort-broadcasting $(y, x)$ then becomes true, which means MSS will sent abort broadcasting to $\mathrm{MH}$.

\section{- Home Server (HS) level}

\section{Register}

At registration stage, $\mathrm{HS}$ sends the pages in the $\mathrm{MH}$ profile, marks the $\mathrm{MH}$ as a valid reader of those pages, and notes where to contact the $\mathrm{MH}$.

$$
\text { r1: holds }(\operatorname{sent}(z, y), \operatorname{res}(\operatorname{send}-\operatorname{page}(z, y), s)) \leftarrow \operatorname{holds}(\operatorname{proxy}(y, x), s)
$$

If $\operatorname{proxy}(y, x)$ is true, action send-page $(z, y)$ will take place, then $\operatorname{sent}(z, y)$ becomes true.

r2: holds $(M H-\operatorname{marked}(z), \operatorname{res}(\operatorname{mark}-M H(z), s)) \leftarrow \operatorname{holds}(\operatorname{sent}(z, y), s)$

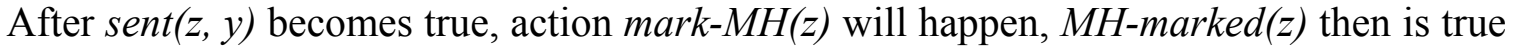
as well.

\section{Start transaction}

After $\mathrm{MH}$ requests a query or update transaction and MSS submits this transaction request to HS, the HS starts the transaction. In update transaction situation, MSS submits transaction only when lock is available.

$$
\text { r3: holds(trans-start(z), res(do-trans(z), s)) } \leftarrow \text { holds(trans-submitted(y), s) }
$$

After transaction request is submitted by MSS, i.e., trans-submitted(y) is true, action do$\operatorname{trans}(z)$ happens, and trans-start(z) then becomes true, the transaction starts. 


\section{Sleep}

The sleeping $\mathrm{MH}$ process may not return (e.g., MH dies, leaves cell), in this case the proxy may wait around aimlessly. To remedy this problem, the MH status is sent to HS after a system-specific amount of time and the proxy process is killed. The decision is made based on $\mathrm{MH}$-sleep-time or time-MH-contacted-proxy.

$$
\begin{aligned}
& \text { r4: holds(proxy-killed(z, y), res(kill-proxy }(z, y), s)) \leftarrow \\
& \text { holds(timeout }(x), s) \text {, holds(vol-slept }(x), s) \\
& \text { r5: holds(proxy-killed(z, y), res(kill-proxy }(z, y), s)) \leftarrow \\
& \text { holds(timeout(x), s), holds(invol-slept(x), s) }
\end{aligned}
$$

If MH goes to sleep, i.e., vol-slept $(x)$ or invol-slept $(x)$ is true, and also timeout $(x)$ is true as well, action kill-proxy $(z, y)$ will happen and $\operatorname{proxy}-k i l l e d(z, y)$ then becomes true.

\section{Move/handoff}

After MH moves to new MSS, the old MSS will flush any dirty pages to HS and new MSS will contact HS to get these dirty pages regarding the MH.

r6: holds(sent(z, y2), res(send-dirtypage $(z, y 2), s)) \leftarrow \operatorname{holds}(\operatorname{proxy}(y 2, x), s)$

After new MSS creates proxy for MH, i.e., $\operatorname{proxy}(y 2, x)$ is true, action send-dirtypage $(z$, $y 2)$ will happen, $\operatorname{sent}(z, y 2)$ then becomes true, the pages is sent to MSS from HS.

\section{Transaction Commit}

According to the two phase commit protocol, if all involved MHs agree to commit the transaction, then the transaction will be committed. If any of them does not agree with commit and want to abort the transaction, then transaction will be aborted. If after a period time (timeout2) the transaction is still not be agreed to be committed, then we use closed world assumption here to assume the transaction won't be committed any more, unknown (not) becomes no $(\neg)$ in this scenario. For example in the case one of the involved $\mathrm{MH}$ has gone to voluntary or involuntary sleep and therefore no commit agreement can be available from that $\mathrm{MH}$ during this time duration. After transaction has been committed or aborted, the HS will send transaction commit or abort notice to MSS.

$$
\text { r7: holds(trans-committed(z), res(commit(z), s)) } \leftarrow
$$

hold(commit-agreed(z), s), holds(trans-start(z), s),not holds(abort-agreed(z), s)

If commit-agreed(z) and trans-start(z) are true, and abort-agreed(z) is not true, i.e. all the parts agree to commit and none of them agrees to abort, then commit action $\operatorname{commit}(z)$ will take place, the transaction is committed, i.e., trans-committed(z) becomes true.

r8: Tholds(trans-committed(z), s) $\leftarrow$ holds(abort-agreed(z), s), holds(trans-start(z), s) If any part agrees to abort, i.e. abort-agreed(z) is true, trans-committed(z) will not be true any more.

$$
\text { r9: -7holds(trans-committed(z), s) } \leftarrow
$$

holds(timeout2(z), s), holds(trans-start(z), s), not holds(trans-committed(z), s)

If transaction commit is not be confirmed, i.e. trans-committed( $z)$ is not holds, and time is over the limit, i.e. holds(timeout2(z), s), the transaction commit will be assumed to be false, i.e. $\rightarrow$ holds(trans-committed(z), s).

$$
\begin{gathered}
\text { r10: holds(commit-noticed(z, y), res(notice-commit }(z, y), s)) \leftarrow \\
\text { hold(trans-committed }(z), s)
\end{gathered}
$$


After transaction commit, i.e. trans-committed(z) becomes true, action notice-commit $(z$, y) will take place to send MSS a commit notice.

r11: holds(abort-noticed(z, y), res(notice-abort(z,y), s)) $\leftarrow \neg$ hold(trans-committed(z), s) After transaction abort, i.e. trans-committed $(z)$ is in false, action notice-commit $(z, y)$ will take place to send MSS a abort notice.

Now, based on the discussion above, we can formally define our transaction model as follows.

Definition: Given language $\mathscr{L}$, a knowledge transaction model on domain $D$ is a pair $\Sigma=(R, I(D))$, where $R$ is the set of knowledge transaction rules imposed above, and $I(D)$ is a finite set of initial facts and rules with respect to domain $D$. We say that a fact $\varphi$ is entailed by $\Sigma$, iff $R \cup I(D) \mid=\varphi$.

In the knowledge transaction model above, $I(D)$ includes initial facts and special rules with respect to domain $D$ only. For example, if we study a knowledge transaction in a investment domain, special rules are the rules related to investment only. For instance, they could be rules for investment risk, profit and fund. Initial facts apparently can be the initial investment status. $\varphi$ can be the fomula like $[\neg] \operatorname{holds}\left(F, \operatorname{res}\left(A_{1}, \operatorname{res}\left(A_{2}, S_{0}\right)\right)\right.$, which should be true in every answer set of $\Sigma$.

\section{A Transaction Example in Mobile Environments}

In this section, we will give an example to explain how to use our logic programming based transaction processing language to describe transaction in the mobile environments. In our example, an initial fact and a finite set of rules with respect to share investment problem domain will be given and specified. The given example discusses a yes scenarios for an update transaction: The $\mathrm{MH}$ requests an update transaction, the transaction is committed on HS using two phase commit protocol. The HS sends commit notice to MSS, MSS then broadcasts the commit result to MH, the MH updates the local knowledge base accordingly. This is yes scenario for an update transaction.

We use our proposed logical programming based transaction processing language $\mathscr{L}$ and model in section 4 as our restriction language and model here by replacing $\mathrm{x}, \mathrm{y}, \mathrm{z}$ with $\mathrm{MH}, \mathrm{MSS}$, and HS. We assume there is a local knowledge base on MH. Let $\mathrm{S}_{0}$ be the initial state, and suppose we are given two initial facts for MH1:

$$
\begin{aligned}
& \text { t1: holds(registered(MSS1, MH1), } \left.S_{0}\right) \leftarrow \\
& \text { t2: holds(profit(share1,MH1), } \left.S_{0}\right) \leftarrow
\end{aligned}
$$

t1 means MH1 has registered in MSS1, t2 means MH1 is profit to invest share1.

There are following rules are introduced here for investment problem domain:

If a sharel has a high profit, low risk, then share 1 can be invested from involved MH's point of view.

$$
\begin{gathered}
\text { i1: holds(invest(share1, } M H i), s) \leftarrow \text { holds(profit(share1, MHi), s), } \\
\text { not hold(risk(share1, MHi), s) }
\end{gathered}
$$


The share1 will not be invested if the risk is high. The cancellation rule is

$$
\text { i2: ᄀholds(invest(share1, MHi), s) } \leftarrow \text { hold(risk(share1, MHi), s) }
$$

The MH1 want to invest share1 based on the rule $i 1, i 2$ and initial fact t2. MH1 requests an update transaction to do the investment and update its local knowledge base accordingly if this investment transaction is committed in HS level. According to $\mathrm{MH}$ level $\mathrm{r} 4$ and $\mathrm{r} 5$ in section 4 , we have

$$
\begin{gathered}
\text { t3: holds(update-requested(MH1), res(write }(M H 1), s)) \leftarrow \\
\text { t4:holds(trans-start(MH1), s) } \leftarrow \text { holds(locked(MH1), res(acquire-lock(MH1), } \\
\text { res(write(MH1), s))), holds(update-requested(MH1), s) }
\end{gathered}
$$

After $\mathrm{MH} 1$ requests an update transaction and the lock has been acquired, the transaction will start.

MSS1 will submit this transaction request to HS. According to MSS level r6 in section 4, we have

$$
\begin{array}{r}
\text { t5:holds(trans-submitted(MSS1), res(submit(MSS1), s)) } \leftarrow \text { holds(locked(MH1), s), } \\
\text { holds(update-required(MH1), s) }
\end{array}
$$

As long as MSS submits the transaction to HS, the HS will start the transaction. According to HS level $\mathrm{r} 3$ in section 4, we have

t6: holds(trans-start(HS), res(do-trans(HS), s)) $\leftarrow$ holds(trans-submitted(MSS1), s)

Then HS will send commit request to all involved MHs for this investment, if all the involved MHs response yes based on the investment rules, the transaction is committed according to the two phase commit protocol. The HS sends commit notice to MSS. According to the HS level $\mathrm{r} 7$ and r10, we have

t7: holds(trans-committed(HS), res(commit(HS), s)) $\leftarrow$ hold(commit-agreed(HS), s), not holds(abort-agreed(HS), s), holds(trans-start(HS), s)

t8: holds(commit-noticed(HS, MSS1), res(notice-commit(HS, MSS1), s)) $\leftarrow$ hold(trans-committed(HS), s)

The MSS1 then broadcasts the commit result to MH1. According to MSS level r14 in section 4 , we have

$$
\begin{gathered}
\text { t9:holds(commit-broadcasting(MSS1, MH1), res(broadcast(MSS1, MH1), s) }) \leftarrow \\
\text { holds(commit-noticed(HS, MSS1), s) }
\end{gathered}
$$

All involved MHs will update the local knowledge base accordingly after the MSS broadcasts the commit result to MHs. According to the MH level r13 in section 4, we have

t10: holds(knowledge-updated(MH), res(update-knowledge(MH), s)) $\leftarrow$

holds(commit-broadcasting(MSS1, MH), s), holds(trans-start(MH), s)

Base on the formal definition given in section 4 , we have $I(D)$ to denote initial facts and rules with respect to investment domain, we have $R$ to denote the set of general knowledge transaction rules. In this example, $I(D)=\{t 1, t 2, i 1, i 2\}$, $R=\{t 3, t 4, t 5, t 6, t 7, t 8, t 9, t 10\}$, therefore we know the fact $\varphi$ is entailed here from $R \cup I(D)$, i.e. $R \cup I(D) \mid=\varphi$, we have $\varphi$ here as following:

$$
\varphi=\left\{\text { holds(trans-committed(HS), res(commit(HS), } S_{0}\right) \text { ), }
$$$$
\text { holds(knowledge-updated(MH), res(update-knowledge } \left.(M H), S_{0}\right) \text { ), }
$$$$
\text { holds(invest(share1, MH1), } S_{0} \text { ) \} }
$$

i.e. the facts that transaction is committed on HS, knowledge base is updated on MHs and share1 is invested on MH1 are entailed in this example. 


\section{Conclusion}

In this paper, we developed and formalized a rule based knowledge transaction model for mobile environments, our model integrated the features of both mobile environments and intelligent agents. We have justified that extended logic programming is a suitable formal specification method to study knowledge transaction in mobile environments. Our knowledge transaction model can be used for knowledge transaction representation, formalization and reasoning in mobile environments. We also demonstrated how our transaction model can be used in practical domains in mobile environments. In the future, we will use this knowledge transaction model to discuss the distributed knowledge transaction processing and multiagent systems in mobile environments.

\section{References}

[1] M. Ahamad, and S. Smith, "Detecting Mutual Consistency of Shared Objects" Proceedings of the IEEE - Conference on Mobile Computing, 1995, pp. 45-50.

[2] L.C. Aiello, and F. Massacci, "Verifying Security Protocols as Planning in Logic Programming", ACM Transactions on Computational Logic, October 2001, pp. 542-580.

[3] C. Baral, Knowledge Representation, Reasoning and Declarative Problem Solving, Cambridge University Press, 2003.

[4] C. Baral, and M. Gelfond, "Logic Programming and Knowledge Representation", Logic Programming, 1994, pp. 73-148.

[5] D. Barbara, and T. Imielinski, "Sleepers and Workaholics: Caching Strategies in Mobile Environments", Proceedings of ACM-SIGMOD 1993 International Conference on Management of Data, May 1994, pp. 1-13.

[6] B. Blaybrook, "On Line Transaction Processing Systems", John Wiley \& Sons, 1992

[7] W.K. Chan, and J. Chen, "Serializability and Epsilon Serializability in a Mobile Environment", Proceedings of seventeenth IASTED International Conference in Applied Informatics, 1999, pp.273-297.

[8] M. Gelfond, and V. Lifschitz, "The Stable Model Semantics for Logic Programming", Proceedings of the fifth International Conference in Logic Programming, 1988, pp. 10701080.

[9] M. Gelfond, and V. Lifschitz, "Logic Programs with Classical Negation", Proceedings of Seventh international Conference in Logic Programming, 1990, pp. 579597.

[10] M. Gelfond, and V. Lifschitz, "Classical Negation in Logic Programs and Disjunctive Databases", New Generation Computing, 1991, pp. 365-385.

[11] J. Gray, and A. Reuter, Transaction Processing: Concepts and Techniques, Morgan Kaufman, 1993.

[12] S. Hanks, and D. McDermott, "Nonmonotonic Logic and Temporal Projection", Artificial Intelligence, 1987, pp. 379-412.

[13] T. Imielinski, and H.F. Korth, Mobile Computing, Kluwer Academic Publishers, 1996. 
[14] T. Komiya, T. Enokido, and M. Takizawa, "Mobile Agent Model for Transaction Processing on Distributed Objects", information Sciences, 2003, pp.1-16.

[15] R. Kowalski, and F. Sadri "Logic Programs with Exceptions", Logic Programming: Proceedings of the Seventh International Conference, 1990, pp.598-613.

[16] S. Levi, and A. Agrawala, Real Time System Design, McGraw-Hill, 1990.

[17] D. Milojicic, “Mobile Agent Applications”, IEEE Concurrency, 1999, pp. 80-90.

[18] N. Mirghafori, and A. Fontaine, "A Design for File Access in a Mobile Environment", Proceedings of the IEEE - Conference on Mobile Computing, 1995, pp. 57-61.

[19] L. Pereira, and J. Alferes, "Well Founded Semantics for Logic Programs with Explicit Negation", Proceedings of European Conference on AI, 1992.

[20] L. Pereira, and J. Alferes, "Nonmonotonic Reasoning with Logic Programming", Journal of Logic Programming, 1993, pp. 227-264.

[21] G. Wagner, "Logic Programming with Strong Negation and Inexact Predicates", Journal of Logic and Computation, 1991, pp. 835-861.

[22] G. Weiss, Multiagent Systems: A Modern Approach to Distributed Artificial Intelligence, The MIT Press, Cambridge, Massachusetts, 1999.

[23] M. Wooldridge, An Introduction to Multiagent Systems, John Wiley \& Sons, LTD, 2002. 